\title{
APLICATIVO PARA TOMADA DE DECISÃo NA AERAÇÃO DE GRÃOS ARMAZENADOS
}

\section{Leandro Levate Macedo ${ }^{1}$ \\ Cintia da Silva Araújo ${ }^{2}$ \\ Luís César da Silva ${ }^{3}$}

Resumo: Os produtos agrícolas são os principais alimentos consumidos pela população mundial, inclusive pelos brasileiros. Diante de uma elevada produção anual de grãos, o controle das condições do ecossistema associado à armazenagem de grãos demanda a condução da operação de aeração em momentos propícios de tal forma evitar a supersecagem ou a deterioração fúngica do produto. Dessa forma, o objetivo deste trabalho foi desenvolver um aplicativo computacional para auxiliar a tomada de decisão no processo de aeração. O aplicativo desenvolvido, "GrãosAera", procede os cálculos das propriedades psicrométricas do ar ambiente, aplicado e intragranular e do teor de umidade de equilíbrio. Procedendo a análise dos valores calculados o aplicativo gera recomendações que fundamentem a tomada de decisão.

Palavras-chave: Psicrometria; Atividade de água; Umidade de equilíbrio.

1 Programa de Pós-graduação em Ciência e Tecnologia de Alimentos / Universidade Federal do Espírito Santo, Brasil. leandrolevate@hotmail.com, araujo.s.cintia@gmail.com.

1 Professor no Programa de Pós-graduação em Ciência e Tecnologia de Alimentos, Universidade Federal do Espírito Santo, Brasil. silvaluisc@yahoo.com.

\footnotetext{
${ }^{1}$ Programa de Pós-graduação em Ciência e Tecnologia de Alimentos/Universidade Federal do Espírito Santo, Brasil. E-mail: leandrolevate@hotmail.com.

2 Programa de Pós-graduação em Ciência e Tecnologia de Alimentos/Universidade Federal do Espírito Santo, Brasil. E-mail: araujo.s.cintia@gmail.com.

3 Professor no Programa de Pós-graduação em Ciência e Tecnologia de Alimentos/Universidade Federal do Espírito Santo, Brasil. E-mail: silvaluisc@yahoo.com.
} 